\title{
Efectos de la terapia cognitivo-conductual sobre la depresión en personas mayores institucionalizadas
}

\author{
Alicia Sales, Alicia Pardo Atiénzar, Teresa Mayordomo, Encar Satorres-Pons y Juan C. Meléndez \\ Universidad Autónoma de Madrid, Madrid, España
}

\begin{abstract}
Resumen: Numerosos estudios han puesto de manifiesto la alta prevalencia de la depresión, cuyas cifras son todavía mayores en la vejez, especialmente en personas mayores institucionalizadas. Ello hace que encontrar un tratamiento eficaz para combatirla sea esencial. Este estudio tiene como objetivo comprobar que la aplicación de una terapia de reestructuración cognitiva en ancianos deprimidos institucionalizados sin deterioro cognitivo es efectiva para la mejora de la depresión así como en diferentes variables consideradas. Para ello, se realizó un estudio experimental aleatorizado con dos grupos de análisis (experimental y control), en el que, tras la aplicación de dicha terapia, se evaluó el cambio sufrido en las diferentes variables psicológicas medidas en una muestra de 8 ancianos deprimidos institucionalizados sin daño neurológico de la provincia de Albacete. Los resultados encontrados, demostraron resultados positivos de esta terapia para reducir la depresión en ancianos deprimidos institucionalizados sin deterioro cognitivo, así como en la mejora las diferentes variables consideradas.
\end{abstract}

Palabras clave: Depresión; personas mayores; terapia cognitiva; reestructuración cognitiva.

\section{Effects of cognitive behavioral therapy on depression in institutionalized elderly}

\begin{abstract}
Numerous studies have shown the high prevalence of depression, figures being even higher in the elderly, especially in institutionalized elderly. Thus finding an effective treatment is essential. This study aims to verify whether the application of cognitive restructuring therapy to depressed institutionalized elderly without cognitive impairment is effective for improving depression and other different variables. A randomized experimental study with two groups (experimental and control) was performed. After applying the therapy, we evaluated the change in the different psychological variables in a sample of 8 depressed institutionalized elderly without neurological damage in the province of Albacete. The results were positive, reducing depression in depressed institutionalized elderly without cognitive impairment, as well as improving the different variables considered.
\end{abstract}

Keywords: depression; elderly; cognitive therapy; cognitive restructuring.

\section{Introducción}

Durante el envejecimiento, las personas mayores deben hacer frente a situaciones relacionadas con riesgos en la salud, pérdida de seres queridos o aumento de la dependencia, situaciones que se evaluarán como daño o pérdida (Martin, Kliegel, Rott, Poon, \& Johnson, 2008),

Recibido: 14 de octubre de 2014; aceptado: 15 de marzo de 2015. Correspondencia: Alicia Sales Galán, Dpto. de Psicología Evolutiva y de la Educación, Facultad de Psicología, Universidad de Valencia, Av. Blasco Ibáñez 21, 46010 Valencia, España. Correo-e: alicia.sales@, uv.es lo que les hace más vulnerables a la depresión, baja autoestima, mayor ansiedad y problemas emocionales.

La prevalencia de síntomas depresivos de adultos mayores en la población general se estima en un 10-15\% (Steffens, Fisher, Langa, Potter y Plassman, 2009), prevalencia que en personas mayores institucionalizadas aumenta hasta un 46,5\% (Damian, Pastor-Barriuso y Valderrama-Gama, 2010). Esta alta prevalencia está, en parte, causada por la confrontación con acontecimientos vitales negativos, como experiencias de pérdida, enfermedades o discapacidad (Bohlmeijer, Westerhof y Emmerik-de Jong, 2008; Kraaij y Wilde, 2001), siendo, la incapacidad para detectar oportunidades ante la adversi- 
dad, un importante mediador entre los eventos negativos y la depresión (Davis, Wortman y Lehman, 2000). Además, según las personas van envejeciendo se produce un incremento de las preocupaciones, especialmente aquellas referidas a la familia y en relación a la salud, concretamente preocupaciones sobre la posibilidad de llegar a perder la independencia funcional (Benítez, de Trocóniz, Montorio, Baltar, y González, 2003).

Diferentes estudios han mostrado que las intervenciones psicológicas son eficaces en el tratamiento de los trastornos depresivos en los adultos (Cuijpers, van Straten, Warmerdam y Smits, 2008), mostrando además una mayor eficacia de este tipo de terapias psicológicas frente a las terapias farmacológicas. De las diferentes intervenciones psicológicas, la Terapia Cognitivo-Conductual (TCC) es un método de intervención que combina e integra los modelos cognitivo y conductual en una única terapia (Izal, Márquez, Losada, Montorio y Nuevo, 2003) y su efectividad ha sido mostrada a través de diferentes estudios en diferentes trastornos. Con respecto a los trastornos del estado de ánimo, principalmente el trastorno depresivo, en la literatura encontramos diferentes estudios de metaanalisis que muestran efectos positivos sobre la terapia de TCC en la depresión (Peng, Huang, Chen \& Lu, 2009), incluso mostrando resultados a largo plazo (Krishna et al. 2011). Esta técnica de intervención parte de la idea de que la depresión es la consecuencia de una combinación de déficit en las habilidades de afrontamiento, problemas con la regulación emocional y una perspectiva extremadamente negativa del mundo y de la capacidad de la propia persona para actuar adecuadamente en él (Romero Gamarra, 2006).

Este último aspecto relacionado con los pensamientos irracionales y negativos, son un elemento central de la depresión, de modo que la modificación y reestructuración de los mismos es un aspecto crucial dentro de este tipo de trastornos. Dentro de la TCC, la terapia cognitiva parte de la idea de que la depresión es el resultado de una alteración en el procesamiento de información como consecuencia de la activación de emociones o creencias negativas (Vázquez et al, 2002). Existen diferentes autores que defienden esta orientación, si bien las teorías más relevantes en la literatura han sido la Terapia Racional-Emotiva-Conductual (Ellis y Dryden, 1997) y la Terapia Cognitiva para la Depresión (Beck, 1979). Sin embargo, y dada su eficacia, actualmente, el número de estudios relacionados con la aplicación de intervenciones cognitivas en personas mayores es reducido.

Por otra parte, es bien sabido que existen diferencias entre las personas mayores institucionalizadas y no institucionalizadas respecto a variables relacionadas con el envejecimiento con éxito y la calidad de vida como son las AVD y el bienestar (Meléndez, Navarro, Sales y Mayordomo, 2012), siendo estas diferencias desfavorables para el grupo institucionalizado. Tal y como señalan estos autores, y partiendo de la idea de que el envejecimiento es un suceso vital que puede modificar el bienestar, la institucionalización parece disminuir en mayor medida éste, debido posiblemente a la pérdida de control sobre las situaciones de su vida y de habilidad para manejarlas, lo que da lugar a una reducción del estado emocional de la persona institucionalizada. Además, y teniendo en cuenta las principales causas de la institucionalización (la pérdida del cónyuge, la no existencia de hijos o de familiares con disponibilidad para el cuidado, etc.), éstos son factores relacionados con la falta de apoyo social, aspecto fundamental para enfrentarse durante el envejecimiento a situaciones estresantes (Meléndez, Tomás y Navarro, 2008), y con la sintomatología depresiva. Es por ello, que el avance en el conocimiento sobre el diagnóstico adecuado e intervención del estado de ánimo en la fase final de la vida reviste gran importancia (García-Soriano y Martín, 2008).

De este modo, y como objetivo principal de este estudio, se pretende comprobar la efectividad de una terapia de reestructuración cognitiva en la sintomatología depresiva así como a través de diferentes variables clínicas, en personas mayores deprimidas e institucionalizadas, sin deterioro cognitivo. A partir de este objetivo, se plantea que se obtendrán diferencias entre los grupos tras la aplicación de la terapia, proponiéndose las siguientes hipótesis: (a) Los niveles de depresión, estrés y ansiedad del grupo tratamiento disminuirán significativamente; (b) Se observará una mejora significativa de la autoestima, la resiliencia y la satisfacción del grupo tratamiento; c. En relación al apoyo, se apreciará un aumento significativo del apoyo emocional, del apoyo afectivo, del apoyo global y del apoyo en la interacción social positiva del grupo tratamiento.

\section{Método}

\section{Participantes}

Para la realización de la investigación se obtuvo una muestra total de ocho sujetos de una residencia de mayores de Mahora (Albacete), de edades comprendidas entre 85 y 93 años, situándose la media de edad del grupo en 89 años. Se realizó un estudio experimental aleatorizado con dos grupos de análisis, un grupo experimental $(n=$ $4)$ y un grupo control $(n=4)$, los cuales fueron evaluados en dos periodos de tiempo diferentes (pre y post tratamiento). 
En relación a los criterios de inclusión para este estudio, era requisito que los participantes de ambos grupos fueran mayores de 65 años y que presentasen sintomatología depresiva, con una puntuación igual o mayor a 11 en la Escala de Depresión Geriátrica (GDS) de Yesavage et al (1983); y una puntuación igual o mayor a 10 en las Escalas de Depresión, Ansiedad y Estrés (DASS) de Lovibond y Lovibond (1995).

Respecto a los criterios de exclusión fueron desestimados para este trabajo aquellos sujetos que tuviesen deterioro cognitivo medido a través del Mini Examen Cognoscitivo (MEC) de Lobo, Saz y Marcos (2002) (desechándose aquellos sujetos que tuviesen una puntuación igual o menor a 23), y aquellos sujetos que presentaran historial de enfermedad neurológica grave, trastorno psiquiátrico o enfermedad sistémica grave (a excepción del trastorno depresivo), historia de abuso de sustancias de acuerdo con el DSM-IV-TR, diagnóstico de demencia según los criterios del DSM-IV-TR (APA, 2002), uso crónico de medicación psicoactiva o hipnótica, y analfabetismo.

Aquellos participantes que cumplían con los criterios firmaron el consentimiento informado para la participación en el estudio. En relación a las características de cada grupo, a continuación, se presentan en la Tabla 1 sus principales características sociodemográficas.

Tabla 1. Frecuencias de las características sociodemográficas de los grupos

\begin{tabular}{lcc}
\hline & $\begin{array}{c}\text { Grupo } \\
\text { tratamiento }\end{array}$ & $\begin{array}{c}\text { Grupo } \\
\text { control }\end{array}$ \\
\hline Edad & $89.5(2.6)$ & $88.5(3.4)$ \\
Genero (H/M) & $1 / 3$ & $2 / 2$ \\
Estado civil (casado/viudos) & $0 / 4$ & $1 / 3$ \\
Escolaridad (sin estudios/primaria) & $2 / 2$ & $2 / 2$ \\
Número de hijos & $1.75(0.95)$ & $1.5(1.2)$ \\
Nivel cognitivo (MEC) & $25.75(0.96)$ & $26.25(1.7)$ \\
\hline
\end{tabular}

Finalmente, al establecer comparaciones entre los grupos (tratamiento vs control), se corroboró la homogeneidad de estos, pues se observó que no diferían en edad $(89.5$ vs $88.5 ; t(6)=.463$, n.s. $)$, género $\left(c^{2}(1)=.50\right.$, n.s. $)$, estado civil $\left(\mathrm{c}^{2}(1)=4.51\right.$, n.s. $)$, estudios $\left(\mathrm{c}^{2}(1)=\right.$ .001 , n.s.), número de hijos (1.75 vs $1.50 ; t(6)=.311$, n.s.) y en nivel cognitivo $(25.75$ vs $26.25 ; t(6)=.632$, n.s.).

\section{Instrumentos}

Previo al periodo de aplicación del tratamiento, se realizó la evaluación de los participantes. En primer lugar se registraron datos sociodemográficos de cada par- ticipante: sexo, edad, estado civil actual, nivel de escolaridad alcanzado y número de hijos en el caso de que tuviera alguno. Posteriormente se aplicaron los instrumentos de evaluación. La aplicación de los instrumentos se llevó a cabo, de forma individual con un tiempo aproximado de 45 minutos por participante. La aplicación de los mismos se realizó antes y después de la intervención.

Para descartar la existencia de un posible deterioro cognitivo, se administró el Mini Examen Cognoscitivo (MEC) de Lobo, Saz y Marcos (2002). Este instrumento de "screening" de deterioro cognitivo consta de 11 ítems en los que se valoran 8 áreas cognitivas: orientación espacio-temporal, memoria de fijación y reciente, atención-concentración y cálculo, lenguaje comprensivo y expresivo, pensamiento abstracto y construcción visoespacial. La puntuación máxima es de 30 puntos, que se obtienen mediante la suma de las puntuaciones de cada ítem y los ítems están agrupados en 5 apartados que comprueban orientación, memoria inmediata, atención y cálculo, recuerdo diferido, lenguaje y construcción. El punto de corte para demencia se establece habitualmente en 24 puntos, las puntuaciones entre 23-21 indican una demencia leve, entre 20-11 una demencia moderada y menor de 10 de una demencia severa. Es una prueba muy utilizada en personas mayores por contar con valores muy altos de fiabilidad (Lobo, Escobar, Ezquerra y Seva Díaz, 1980).

Y para valorar la posible existencia de dependencia física y repercusión de las actividades básicas de la vida diaria, se pasó el Índice de Barthel (Mahoney y Barthel, 1965). Este índice evalúa la independencia física de las personas mayores para realizar 10 actividades básicas de la vida diaria: comer, lavarse, vestirse, arreglarse, deposiciones, micciones, uso del retrete, trasladarse, deambular y uso de escalones. La puntuación asignada a cada actividad se basa en el tiempo y en la cantidad de ayuda física requerida por el sujeto, de tal manera que se le concede 0 puntos cuando hay total dependencia, 5 puntos cuando necesita ayuda, 10 puntos cuando no requiere ayuda pero es necesaria la supervisión y 15 puntos cuando no necesita ni ayuda ni supervisión. A menor puntuación, más dependencia; y a mayor puntuación, más independencia, siendo una puntuación entre 0-20 equivalente a una dependencia total; 21-60 dependencia severa; 61-90 dependencia moderada; 91-99 dependencia escasa; 100 independencia. En lo referente a las propiedades psicométricas, esta prueba ha demostrado tener buenas garantías de fiabilidad y validez (Cid-Ruzafa y Damián-Moreno, 1997).

A continuación, los participantes cumplimentaron diferentes escalas y tests que se indican abajo con el fin de evaluar diversas variables de interés de cara a la aplicación de la intervención de reestructuración cognitiva. 
Escalas de Depresión, Ansiedad y Estrés (DASS; Lovibond y Lovibond, 1995), forman parte del cuestionario DASS, compuesto por 42 ítems que miden los estados emocionales negativos de la depresión, la ansiedad y el estrés a través de tres escalas. Cada una éstas contiene 14 ítems, divididos a su vez en diferentes subescalas. La escala de estrés evalúa activación y tensión persistentes con facilidad de llegar a causar frustración, es decir, dificultad para relajarse, activación nerviosa y facilidad para agitarse, para irritarse o para impacientarse. La escala de ansiedad se centra en identificar los síntomas somáticos y subjetivos de miedo, esto es, activación autónoma, efectos musculares, ansiedad situacional y experiencia subjetiva de ansiedad. Por último, la escala de depresión evalúa disforia, desesperanza, desvalorización de la vida, auto-desaprobación, falta de interés o de implicación, anhedonia e inacción. Las respuestas se dan en una escala tipo Likert de 4 puntos, que van desde " 0 " que indica nada aplicable a mí hasta "3" que significa muy aplicable a mí o aplicable la mayor parte del tiempo. Tienen una excelente consistencia interna y estabilidad temporal (Brown, Chorpita, Korotitsch y Barlow, 1997), además, se ha demostrado su validez en personas de avanzada edad (Gomez, Summers, Summers, Wolf y Summers, 2013).

Escala de Depresión Geriátrica de Yesavage (GDS; Yesavage et al, 1983). Construida para detectar síntomas de depresión en personas mayores. Es una prueba fácil de administrar (Yesavage et al, 1983), la cual consta de 30 preguntas de respuesta dicotómica si/no cuyo contenido está centrado en aspectos sobre irritabilidad, pensamientos angustiosos, afecto, aislamiento, juicios negativos e inactividad. Ha demostrado ser un instrumento útil de evaluación de la depresión en personas mayores con capacidades cognitivas intactas (Izal, Montorio, Nuevo y Pérez-Rojo, 2007).

Escala de Autoestima (SES; Rosenberg, 1965). Fue diseñada para ser una medida unidimensional de autoestima, considerando ésta como un sentimiento positivo o negativo hacia uno mismo, el cual se construye a través de la valoración de las propias características. Por ello, esta escala es una de las pruebas más utilizadas a la hora de medir autoestima. Está compuesta de 10 ítems, la mitad expresados en forma positiva y la otra mitad en forma negativa, representados en una escala tipo Likert de 4 puntos que oscila desde "1" (muy de acuerdo) hasta " 4 " (muy en desacuerdo). Esto permite obtener una puntuación mínima de 10 y máxima de 40 , a través de su sencilla y rápida aplicación, donde una mayor calificación indicará una mayor autoestima del sujeto. Su estructura, basada en un único factor, ha mostrado buenos niveles de consistencia interna y estabilidad temporal (Martín-Albo, Núñez, Navarro y Grijalvo, 2007).
Escala de Satisfacción de Filadelfia (PGC). Diseñada por Lawton en 1972 (Lawton, 1975), evalúa la satisfacción vital, considerada como un concepto multidimensional del bienestar psicológico de las personas mayores con tres factores principales: agitación, actitud hacia el propio envejecimiento e insatisfacción con la soledad. Se ha usado esta escala por ser una herramienta útil en personas mayores con adecuadas propiedades psicométricas. La versión utilizada en este trabajo consta de 16 preguntas de respuesta dicotómica si/no. La escala ha sido adaptada y validada al español con una fiabilidad muy satisfactoria (Montorio, 1990).

Cuestionario Brief Resilient Coping Scale (BRCS; Sinclair y Wallston, 2004). Mide el nivel de resiliencia, entendida ésta como un proceso dinámico que implica una adaptación positiva del sujeto en un contexto de adversidad y estrés significativos. Consta de 4 ítems representados mediante una escala tipo Likert de 5 puntos, donde se considera el valor " 1 " cuando el ítem no refleja nada la reacción típica de la persona y el valor " 5 " cuando el ítem refleja la forma habitual en la que ésta responde a los problemas. A pesar de su brevedad, esta prueba ofrece una gran estabilidad y una adecuada consistencia interna (Tomás, Meléndez, Sancho y Mayordomo, 2012).

Cuestionario MOS de Apoyo Social (MOS; Sherbourne y Stewart, 1991), evalúa el apoyo social del sujeto a través de diferentes dimensiones: apoyo emocional (comprensión empática y afecto), apoyo instrumental (ayuda material), apoyo afectivo (expresiones de amor), apoyo en interacción social positiva (distracción y diversión con otras personas) y apoyo social global (incluye todas las anteriores). Consta de 20 ítems medidos todos ellos, a excepción del primero, en una escala de respuesta tipo Likert de 5 puntos, donde " 1 " indica nunca y " 5 " siempre, de tal manera que una mayor puntuación revelará un mayor nivel de apoyo social percibido por el sujeto. Al analizar sus cualidades psicométricas, éstas mostraron fiabilidad y estabilidad en el tiempo (Costa Requena, Salamero y Gil, 2007; Sherbourne y Stewart, 1991).

\section{Procedimiento}

El programa de intervención estuvo fundamentado en terapia de reestructuración cognitiva, la cual se aplicó en dos sesiones semanales de una hora durante cuatro semanas, con un total de ocho sesiones. Todas las sesiones fueron grupales. A continuación se describen las sesiones.

Sesión 1: la terapia da comienzo con la presentación del programa, objetivos, número de sesiones y duración aproximada. Seguidamente se presentan el terapeuta y 
los participantes con la finalidad de crear un clima de confianza y de seguridad. El resto de la sesión se destina a que cada uno de los sujetos comente algún problema que le inquiete, de esta manera, al compartir sus preocupaciones da la posibilidad de que se den cuenta de que tener problemas es algo normal y que todos los tenemos.

Sesión 2: Se les da una explicación teórica de la "trampa" de la depresión, como comienza y cómo se desarrolla y porque se mantiene, y de esta forma se da sentido al problema. La conceptualización es en sí terapéutica ya que es una forma de comprender, predecir y normalizar los problemas. Se finaliza la sesión con una elaboración del listado de problemas.

Sesión 3: Se explican qué son las creencias irracionales, sus tipos y se les enseña a identificarlas, pues todos las usamos sin darnos cuenta, y no nos ayudan a resolver nuestros problemas. Para practicarlas, aprenderlas e interiorizarlas mejor se plantean al final de la sesión ejercicios grupales e individuales.

Sesión 4: Se explica cómo funciona el modelo $A B C$ y se plantean diversos ejemplos para una mejor comprensión del mismo. A continuación se plantean ejercicios para practicar el modelo $\mathrm{ABC}$ de forma grupal.

Sesión 5: Se comenta la relación existente entre las creencias irracionales y el modelo $\mathrm{ABC}$ y se practica con los propios problemas. Como ejercicio, han de identificar los pensamientos ante sus problemas personales, para practicar su detección así como a registrarlos.

Sesión 6: Se les explica cómo debatir los pensamientos a través de la discusión cognitiva de Beck y Ellis. Con esto se pretende sustituir la idea irracional por una racional a través de un debate dialectico donde a través de preguntas, se le pide a la persona y se le reta a demostrar la objetividad de los pensamientos y creencias. Se ponen diversos ejemplos de debate de pensamientos para su comprensión

Sesión 7: Se pone en práctica el debate con los propios pensamientos registrados en sesiones anteriores y se les enseña el último paso: la búsqueda de un pensamiento alternativo y objetivo a la vez que realista, frente al antiguo pensamiento irracional.

Sesión 8: En la última sesión, se generan reflexiones finales sobre el tema, resolución de dudas, se realiza una valoración de la terapia y se comenta la utilidad de la misma, se anima a los participantes a continuar con la misma actitud y su entrenamiento constante para su interiorización y se da paso a la despedida.

\section{Tratamiento estadístico de los datos}

Dado el tamaño muestral, los análisis se realizaron mediante pruebas no paramétricas. Se aplicó la U
Mann-Whitney para la comparación entre grupos en las medidas tanto pre como postest y Wilcoxon para el análisis del cambio entre el tiempo 1 y el tiempo 2 para cada grupo, tomado de forma independiente. Todos estos análisis se realizaron a través del programa estadístico SPSS 20.

\section{Resultados}

A continuación se exponen los resultados de las variables analizadas, presentándose las diferencias estadísticamente significativas entre los grupos tratamiento y control al comparar sus medidas en el pretest y el postest, así como la existencia de cambios significativos del tiempo 1 al tiempo 2 dentro de cada grupo.

En relación al cuestionario DASS las puntuaciones del pretest no mostraron diferencias significativas entre los grupos tratamiento y control en depresión (Mann-Whitney $z=.726$; n.s.), ansiedad ( $z=.289$; n.s.) y estrés $(z=.438$; n.s. $)$ ni en los postest para depresión $(z$ $=1.44$; n.s. $)$, ansiedad $(z=1.16$; n.s. $)$ y estrés $(z=1.16$; n.s.).

El análisis de las diferencias entre las puntuaciones tomadas en el tiempo 1 y el tiempo 2 para el grupo tratamiento, mostraron diferencias significativas para la variable depresión (Wilcoxon $z=2.11 ; p=.048$ ), ansiedad $(z=2.21 ; p=.034)$ y estrés $(z=1.05 ; p=.034)$, mientras que el grupo control, no se observo la existencia de cambio significativo ni para la depresión $(z=.816$; n.s. $)$, ni tampoco para la ansiedad $(z=1.41 ;$ n.s. $)$ y estrés $(z=$ 1.84; n.s.).

A la hora de estimar los resultados obtenidos en la evaluación de la sintomatología depresiva mediante la escala GDS, no se observan diferencias estadísticamente significativas entre los grupos tratamiento y control al comparar sus medidas pretest (Mann-Whitney $z=.296$; n.s.) ni para las postest ( $z=1.46$; n.s.). Al comparar las puntuaciones entre el tiempo 1 y el tiempo 2, los resultados señalan diferencias significativas para el grupo tratamiento (Wilcoxon $z=.54 ; p=.038$ ), mientras que el grupo control no modifica sus puntaciones $(z=.577$; n.s.).

En la valoración de la autoestima, la satisfacción vital y la resiliencia, al comparar las medias de ambos grupos en el pretest no se hallaron diferencias significativas en ninguna de las variables (Mann-Whitney $z=.145$; n.s.; $z=.500 ;$ n.s.; $z=.145$; n.s.), ni tampoco al comparar las puntuaciones postest $(z=.1 .47$; n.s.; $z=2.20$; n.s.; $z=1.75$; n.s.).

El análisis de las diferencias entre los tiempos para cada grupo analizado de forma independiente, en grupo tratamiento señalo que diferencias significativas en auto- 
estima (Wilcoxon $z=1.84 ; p=.046$ ), en satisfacción vital $(z=1.89 ; p=.039)$ y en resiliencia $(z=1.85 ; p=$ .036), mientras que el grupo control no se observaron diferencias significativas en ninguna de las tres variables $(z=.577$; n.s.; $z=.1 .01 ;$ n.s.; $z=.477$; n.s. $)$.

Por último y en relación a la prueba MOS, no se obtuvieron diferencias significativas en las comparaciones pretest para apoyo emocional (Mann-Whitney $z=.730$; n.s.), apoyo instrumental ( $z=.150$; n.s.), apoyo en interacción social $(z=.195 ;$ n.s. $)$, apoyo afectivo $(z=.122$; n.s.), ni apoyo global $(z=.292$; n.s.); del mismo modo las medidas postest tampoco mostraron diferencias apoyo emocional ( $z=1.16$; n.s.), apoyo instrumental ( $z=.132$; n.s.), apoyo en interacción social ( $z=1.46$; n.s.), apoyo afectivo $(z=.146$; n.s. $)$, ni apoyo global $(z=.343$; n.s. $)$.

$\mathrm{Al}$ analizar las diferencias de las puntuaciones tomadas en el tiempo 1 y el tiempo 2 dentro de cada grupo, el grupo tratamiento sólo mostró un cambio significativamente marginal en apoyo en interacción social (Wilcoxon $z=1.84 ; p=.056$ ), mientras que para el resto de las variables del MOS no se mostraron cambios (apoyo emocional: $z=1.46$; n.s.; apoyo instrumental: $z=.736$; n.s.; apoyo afectivo: $z=.577$; n.s.; y apoyo global: $z=$ 1.56; n.s.). Del mismo modo el grupo control no mostró cambio significativo.

En la Tabla 2 se incluyen las medias del grupo de tratamiento y del grupo de control en el tiempo 1 y el tiempo 2 en cada una de las variables analizadas.

Tabla 2. Medias de los grupos tratamiento y control en los tiempos 1 (T1) y 2 (T2).

\begin{tabular}{lrrrr}
\hline & \multicolumn{2}{c}{$\begin{array}{c}\text { Grupo } \\
\text { Tratamiento }\end{array}$} & \multicolumn{2}{c}{$\begin{array}{c}\text { Grupo } \\
\text { Control }\end{array}$} \\
\cline { 2 - 5 } & \multicolumn{1}{c}{ T1 } & \multicolumn{1}{c}{ T2 } & \multicolumn{1}{c}{ T1 } & \multicolumn{1}{c}{ T2 } \\
\hline Depresión & 32.25 & 20.25 & 28.50 & 28.00 \\
Ansiedad & 21.00 & 16.50 & 23.25 & 22.75 \\
Estrés & 26.75 & 19.00 & 23.50 & 25.50 \\
Autoestima & 21.75 & 24.75 & 21.75 & 22.00 \\
Satisfacción vital & 3.75 & 7.25 & 4.50 & 4.25 \\
Resiliencia & 2.12 & 2.68 & 2.06 & 2.12 \\
Apoyo emocional & 21.75 & 26.75 & 21.25 & 21.25 \\
Apoyo instrumental & 16.76 & 17.75 & 17.25 & 17.50 \\
Apoyo en interacción social po- & 10.75 & 15.00 & 12.00 & 12.50 \\
$\quad$ sitiva & & & & \\
Apoyo afectivo & 8.75 & 9.00 & 8.50 & 8.50 \\
Apoyo global & 58.00 & 68.50 & 59.00 & 59.75 \\
\hline
\end{tabular}

\section{Discusión}

El objetivo de este trabajo era analizar la efectividad que tiene la aplicación de una terapia de reestructuración cognitiva en personas mayores deprimidas e institucio- nalizadas y sin deterioro cognitivo sobre la reducción de los niveles de depresión, ansiedad y estrés, la mejora de la autoestima, la resiliencia y la satisfacción, y el aumento del apoyo social. Los resultados obtenidos sugieren que la aplicación de una terapia de reestructuración cognitiva en personas mayores ha sido satisfactoria mostrando la utilidad de este tipo terapia.

Con respecto a la variable depresión, tal y como se esperaba, los resultados reflejaron una disminución de ésta en el grupo tratamiento tras la administración de la terapia. La eficacia de las técnicas cognitivas para el alivio de la depresión en personas mayores ha sido mostrada en otros trabajos como el de Laidlaw, Thompson, Gallagher-Thompson y Dick-Siskin (2003). Lo que sucede es que la sintomatología depresiva, activa esquemas cognitivos irracionales y el sujeto percibe la realidad y su entorno de forma negativa y/o desadaptativa. Desde este tipo de terapia, identifica este tipo de pensamientos y aprende a modificar las cogniciones desadaptativas y ver una realidad más empírica que le permite actuar de forma positiva.

En cuanto al estrés, al igual que en trabajos anteriores (Peralta-Ramírez, Robles-Ortega, Navarrete-Navarrete y Jiménez-Alonso, 2009), los resultados muestran una reducción significativa de los niveles de éste en el grupo tratamiento. Los cambios en el estrés pueden estar causados por la disminución de la indefensión e inseguridad ante el futuro, que aumentaba con la percepción de soledad y aislamiento social; y tras la intervención, los sujetos aprecian mayores capacidades para enfrentarse al futuro y a las situaciones de estrés. En relación con este resultado, y al igual que en otros trabajos (Hendriks, Oude Voshaar, Keijsers, Hoogduin y Van Balkom, 2008; Laidlaw et al., 2003), se ha observado una disminución de la variable ansiedad tras la aplicación de la terapia. Los cambios en la ansiedad pueden radicar en la disminución de la agitación de los participantes tras la revaloración de su futuro, el cual es observado con calma y con mayores oportunidades.

En el envejecimiento se producen cambios, especialmente físicos y cognitivos que llevan a las personas a sentirse menos útiles y dañar así la autoestima. La reestructuración cognitiva ha mostrado ser efectiva para la mejora de la autoestima, al igual que en trabajos anteriores (Connors, Johnson y Stuckey, 1984), ya que a través de este tipo de terapia la persona tiene oportunidad de revalorar sus capacidades, dando lugar a nuevos planteamientos más objetivos y realistas, púes a pesar de la edad y de las pérdidas físicas y cognitivas, todavía poseen grandes cualidades.

Del mismo modo, este debate de pensamientos llevado a cabo desde este tipo de terapia para hacer frente a 
los pensamientos irracionales, los cuales bloquean al individuo que ha de hacer frente a una situación de afrontamiento, permite de nuevo reevaluar los pensamientos adoptando una connotación más objetiva que permite lograr la obtención de objetivos frente a la situación de afrontamiento y lograr así una mayor resiliencia. De este modo, se ha visto la efectividad de la reestructuración cognitiva como técnica para incrementar resiliencia (Gil Andino y Cano Vindel, 2001).

Los resultados encontrados en la variable satisfacción mostraron una mejora de ésta en el grupo tratamiento tras la aplicación de la terapia de reestructuración cognitiva. Los resultados obtenidos en el estudio de Sörensen, Pinquart y Duberstein (2002), mostraron que las terapias cognitivo-conductuales que utilizan, entre otras, la técnica de reestructuración cognitiva, son unas de las más efectivas a la hora de incrementar satisfacción. Si atendemos al concepto de calidad de vida tal y como lo describe Yanguas (2006), dentro de sus componentes encontramos las impresiones subjetivas como es la satisfacción, es decir, la percepción que tiene la persona de su funcionamiento psicológico, social y físico. Desde la terapia, el individuo tiene la posibilidad de reflexionar y obtener una valoración más positiva de sí mismo, o al menos más realista, eliminando connotaciones pesimistas e irracionales.

Por último, y en cuanto a los resultados hallados en el apoyo social, no se han obtenido resultados satisfactorios en las dimensiones del apoyo social. En este sentido, se esperaban tales resultados dado que, tal y como indican Meléndez, Tomás y Navarro (2008), la reducción las redes sociales se podría percibir como amenaza, de modo que la capacidad y el modo de adaptación del sujeto van a ser elementos determinantes para superar los posibles cambios. Así mismo, Hernández Lira, Ramírez Beltrán y Díaz Pineda (2011), señalan que respecto a la mejora del apoyo e interacción social, la terapia cognitiva-conductual es efectiva para reestructurar el contexto del sujeto, lograr una mayor adaptación y mejorar las diferentes redes de apoyo social. Es por ello, y de cara a futuros trabajos, que se podría plantear reforzar las sesiones con el objetivo de potenciar esta dimensión para la posible mejora de los resultados, así como el aumento del tamaño de la muestra, ya que ambos aspectos podrían haber causado tales resultados.

En cuanto a las limitaciones del estudio, cabe destacar el pequeño tamaño muestral y la ausencia de una medida de seguimiento para poder comprobar el mantenimiento de los resultados hallados tras la aplicación de la intervención. De cara a líneas futuras se plantea poner en marcha este tipo de terapia en personas mayores sa- nas con un número de sujetos lo suficientemente grande como para poder obtener un mayor rigor metodológico mediante el uso de pruebas paramétricas, con el fin de aumentar el conocimiento sobre la eficacia de esta técnica en este grupo de población, el cual sigue estando todavía muy poco estudiado. Esto permitiría adaptar correctamente las intervenciones a las características y necesidades propias de la muestra considerada en cada caso, lo que implicaría, como consecuencia, un aumento de las garantías terapéuticas de estas intervenciones en este tipo de población. Por otra parte, sería interesante la obtención de una medida de seguimiento para poder corroborar el mantenimiento de la eficacia de la terapia en el tiempo lo que haría aumentar todavía más su rigor científico.

\section{Bibliografía}

American Psychiatric Association (2002). Manual diagnóstico y estadístico de los trastornos mentales, DSM-IV-TR. Barcelona: Masson.

Beck, A. T. (1979). Cognitive therapy and the emotional disorders. New York: Penguin Books.

Bohlmeijer E. T., Westerhof G. J. y Emmerik-de Jong, M. (2008). The effects of integrative reminiscence on meaning in life: Results of a quasi- experimental study. Aging \& Mental Health, 12, 639-646.

Brown, T. A., Chorpita, B. F., Korotitsch, W. y Barlow, D. (1997). Psychometric properties of the Depression Anxiety Stress Scales (DASS) in clinical samples. Behaviour Research and Therapy, 35, 79-89.

Cid-Ruzafa, J. y Damián-Moreno, J. (1997). Valoración de la discapacidad física: el índice de Barthel. Revista Española Salud Pública, 71, 127-137.

Connors, M., Johnson, C. y Stuckey, M. (1984). Treatment of bulimia with brief psychoeducational group therapy. The American Journal of Psychiatry, 141, 1512-1516.

Costa Requena, G., Salamero, M. y Gil, F. (2007). Validación del cuestionario MOS-SSS de apoyo social en pacientes con cáncer. Medicina Clínica, 128, 687-691.

Cuijpers, P., Van Straten, A., Warmerdam,L. y Andersson, G. (2008). Psychological treatment of depression: A meta-analytic database of randomized studies. BMC Psychiatry, 8, 8-36.

Damián, J., Pastor-Barriuso, R. y Valderrama-Gama, E. (2010). Descriptive epidemiology of undetected depression in institutionalized older people. Journal of the American Medical Directors Association, 11, 312-319.

Davis, C. G., Wortman, C. B. y Lehman, D. R. (2000). Searching for meaning in loss: Are clinical assumptions correct? Death Studies, 24, 497-540.

Ellis, A. y Dryden, W. (1997). The practice of rational emotive behavior therapy. New York: Springer publishing company.

García-Soriano, G. y Martín, P. B. (2008). Trastornos del estado de ánimo al final de la vida: ¿desmoralización o depresión? Revista de Psicopatología y Psicología Clínica, 13, 123-133. 
Gil Andino, A., y Cano Vindel, A. (2001). Aplicación de técnicas cognitivo-conductuales en un caso de ansiedad generalizada. http://www.psiquiatria.com/interpsiquis2001/2255. Descargado el 06/02/2014.

Gomez, R., Summers, M., Summers, A., Wolf, A. y Summers, J. J. (2014). Depression Anxiety Stress Scales-21: Factor structure and test-retest invariance, and temporal stability and uniqueness of latent factors in older adults. Journal of Psychopathology and Behavioral Assessment, 36, 308-317.

Hendriks, G. J., Oude Voshaar, R. C., Keijsers, G. P. J., Hoogduin, C. A. L. y Van Balkom, A. J. (2008). Cognitive-behavioural therapy for late-life anxiety disorders: a systematic review and meta-analysis. Acta Psychiatrica Scandinavica, 117, 403-411.

Hernández Lira, N. A., Ramírez Beltrán, B. y Díaz Pineda, C. (2011). Terapia cognitivo-conductual y entrenamiento en habilidades sociales para esquizofrenia. Psiquiatria. com, 15.

Izal, M., Márquez, M., Losada, A., Montorio, I. y Nuevo, R. (2003). Una revisión sobre las intervenciones cognitivo-conductuales en problemas de depresión en la edad avanzada. Revista Española de Geriatría y Gerontología, 38, 34-45.

Izal, M., Montorio, I., Nuevo, R. y Pérez-Rojo, G. (2007). Comparación de la sensibilidad y la especificidad entre diferentes versiones de la Escala de Depresión Geriátrica. Revista Española de Geriatría y Gerontología, 42, 227-232.

Kraaij, V. y de Wilde, E. J. (2001). Negative life events and depressive symptoms in the elderly: A life span perspective. Aging \& Mental Health, 5, 84-91.

Krishna, M., Jauhari, A., Lepping, P., Turner, J., Crossley, D. y Krishnamoorthy, A. (2011). Is group psychotherapy effective in older adults with depression? A systematic review. International Journal of Geriatric Psychiatry, 26, 331-340.

Laidlaw, K., Thompson, L. W., Gallagher-Thompson, D. y Dick-Siskin, L. (2003). Cognitive behaviour therapy with older people. Chichester: John Wiley \& Sons.

Lawton, M. P. (1975). The Philadelphia Geriatric Center Morale Scale: A revision. Journal of Gerontology, 30, 85-89.

Lobo, A., Escobar, V., Ezquerra, J. y Seva Díaz, A. (1980). El Mini-Examen Cognoscitivo. Un test sencillo, práctico, para detectar alteraciones intelectuales en pacientes psiquiátricos. Revista de Psiquiatría y Psicología Médica, 14, 39-57.

Lobo, A., Saz, P. y Marcos, G. (2002). Adaptación del Examen Cognoscitivo Mini-Metal. Madrid: Tea.

Lovibond, S. y Lovibond, P. (1995). Manual for the depression anxiety stress scales. Sydney, Australia: Psychology Foundation of Australia.

Mahoney, F. I. y Barthel, D. W. (1965). Functional evaluation: the Barthel Index. Maryland State Medical Journal, 14, 61-65.

Martin, P., Kliegel, M., Rott, C., Poon, L. W. y Johnson, M. A. (2008). Age differences and changes of coping behavior in three age groups: findings from the Georgia Centenarian Study. The International Journal of Aging and Human Development, 66, 97-114.

Martin-Albo, J., Núñez, J. L., Navarro, J. G. y Grijalvo, F. (2007). The Rosenberg Self-Esteem Scale: Translation and Validation in University Students. The Spanish Journal of Psychology, $10,458-467$.
Meléndez, J. C., Tomás, J. M. y Navarro, E. (2008). Análisis de las redes sociales en la vejez a través de la entrevista Manhein de apoyo social. Salud Pública México. 49, 408-416.

Meléndez, J.C., Navarro, E., Sales, A. y Mayordomo, T. (2012). Efecto moderador de la institucionalización en las actividades de la vida diaria y bienestar en el envejecimiento. Revista Brasileña de Geriatría y Gerontología, 15, 671-680.

Montorio Cerrato, I. (1990). Evaluación psicológica en la vejez: Instrumentación desde un enfoque multidimensional. (Tesis Doctoral) Universidad Autónoma de Madrid, Madrid.

Nuevo, R. Izal, M., Montorio, I., Losada, A. y Márquez, M. (2003). Dimensiones de contenido de preocupación en población de edad avanzada. Revista de Psicopatología y Psicología Clínica, 8, 223-238.

Peng, X. D., Huang, C. Q., Chen, L. J. y Lu, Z. C. (2009). Cognitive behavioural therapy and reminiscence techniques for the treatment of depression in the elderly: A systematic review. The Journal of International Medical Research, 37, 975-982.

Peralta-Ramírez, M. I., Robles-Ortega, H., Navarrete-Navarrete, N. y Jiménez-Alonso, J. (2009). Aplicación de la terapia de afrontamiento del estrés en dos poblaciones con alto estrés: pacientes crónicos y personas sanas. Salud Mental, 32, 251258.

Romero Gamarra, G. (2006). Tratamiento cognitivo-conductual de un caso de depresión en la tercera edad. Revista de Psiquiatría y Salud Mental Hermilio Valdizan, 7, 65-75.

Rosenberg, M. (1965). Society and the adolescent self-image. Princeton: Princeton University Press.

Sherbourne, C. D. y Stewart, A. L. (1991). The MOS social support survey. Social Science \& Medicine, 32, 705-714.

Sinclair, V. G. y Wallston, K. A. (2004). The development and psychometric evaluation of the Brief Resilient Coping Scale. Assessment, 11, 94-101.

Sörensen, S., Pinquart, M. y Duberstein, P. (2002). How effective are interventions with caregivers? An updated meta-analysis. The Gerontologist, 42, 356-372.

Steffens, D. C., Fisher, G. G., Langa, K. M., Potter, G. G. y Plassman, B. L. (2009). Prevalence of depression among older Americans: The aging, demographics and memory study. International Psychogeriatrics, 21, 879-888.

Tomás, J. M., Meléndez, J. C., Sancho, P. y Mayordomo, T. (2012). Adaptation and initial validation of the BRCS in an elderly Spanish sample. European Journal of Psychological Assessment, 28, 283-289.

Vázquez, F. L., Muñoz, R. F. y Becoña, E. (2002). Depresión: Diagnóstico, modelos teóricos y tratamiento a finales del siglo XX. Psicología Conductual, 8, 417-449.

Yanguas, J.J. (2006). Análisis de la calidad de vida relacionada con la salud en la vejez desde una perspectiva multidimensional. Madrid: IMSERSO, Colección estudios: Serie Personas Mayores.

Yesavage, J. A., Brink, T. L., Rose, T. L., Lum, O., Huang, V., Adey, M. y Leirer, V. O. (1983). Development and validation of a geriatric depression screening scale: A preliminary report. Journal of Psychiatric Research, 17, 37-49. 